\title{
Coupled donors in quantum dots: Quantum size and dielectric mismatch effects
}

\author{
J. L. Movilla, A. Ballester, and J. Planelles* \\ Departament de Química Física i Analítica, Universitat Jaume I, Box 224, E-12080 Castelló, Spain \\ (Received 17 February 2009; revised manuscript received 17 April 2009; published 20 May 2009)
}

\begin{abstract}
Spatial and dielectric confinement modulations of the spontaneous emission rates, transition energies, and charge-density distributions of a singly ionized double donor system $\left(D_{2}^{+}\right)$in a spherical quantum dot are calculated within the framework of the effective-mass envelope function approximation. Dipole moments, energy splittings, transition moments, electron-density distributions, and spontaneous emission rates involving bonding and antibonding lowest-lying molecular states are addressed for different dielectric environments, quantum dot radii, and relative locations of the coupled impurities inside the dot. The results indicate that the donor molecule behaves as heteropolar when the spatial confinement breaks the inversion symmetry, which is paralleled by a strong reduction in the excited-state radiative lifetime. Dielectric confinement, acting on a larger length scale than spatial confinement, may recover the bulklike homopolar character when the dot is embedded in a low dielectric constant medium. In the weak spatial confinement regime, dielectric effects can increase the corresponding bulk radiative lifetimes significantly and simultaneously modulate the charge-density distribution.
\end{abstract}

DOI: 10.1103/PhysRevB.79.195319

PACS number(s): 71.55.-i, 73.21.La, 77.22.Ej, 03.67.Lx

\section{INTRODUCTION}

The system built of two coupled semiconductor quantum dots (QDs) containing electrons, holes, or an exciton constitutes one of the simplest solid structures proposed as the functional part in a wide range of device applications, including spintronics, ${ }^{1}$ optoelectronics, ${ }^{2}$ photovoltaics,${ }^{3}$ and quantum information technologies. ${ }^{4,5}$ The widespread interest in this system relies on the ability to manipulate conveniently its molecular properties, such as the energy splitting between the bonding (nodeless) and antibonding (noded) lowest-lying molecularlike states or the spatial distribution of carriers in the system. ${ }^{6}$ In this regard, the asymmetry of the constituent dots (either intrinsic or intentional) has been viewed not as a flaw but as an essential design choice to provide additional control over the system properties. ${ }^{5,7,8}$

Advances in single-dopant fabrication ${ }^{9-11}$ and charge detection $^{12,13}$ have led atomic scale electronics (particularly the field of quantum computing) to pear increasing attention to another molecular system. Namely, the $D_{2}^{+}$system, i.e., the system built of two coupled donor impurities in a semiconductor host in which one of the two excess electrons has been ionized. This system encodes the logical information either on the spin or on the charge degrees of freedom of the remaining excess electron. ${ }^{14-20}$ Initial proposals relied on spin encoding. However, direct detection of a single spin remains today a major challenge. ${ }^{21}$ On the contrary, a fraction of a single electron charge can be easily detected with state-of-the-art single electron-tunneling devices. ${ }^{12,13}$ This readout feasibility, together with the relative simplicity for the manipulation of charge degrees of freedom as opposed to spin, has raised interest in charge qubits, where the logical states are represented by the two lowest-lying electron orbital states positioned at the different donors. All the same, the short coherence times of orbital states severely limit the scalability of charge qubits, ${ }^{16}$ so that mechanisms enhancing coherence would be desirable.

Studies on confined $D_{2}^{+}$systems have been recently addressed in the literature. ${ }^{22-24}$ Particularly, Kang et al. ${ }^{24}$ found that the confinement of the $D_{2}^{+}$system in a spherical QD can greatly enhance the energy differences between the two lowest-lying electron states and more excited states, favoring the identification of the quasi-two-level system required for quantum computation purposes. ${ }^{25}$

Quite a larger amount of work has been done in the study of the electronic structure and related properties of single shallow donors in nanoscale semiconductor heterostructures. ${ }^{26-28}$ In addition to the well-known quantum size effect, the influence of the dielectric confinement (coming from the different dielectric response of the QD and the surrounding medium) on the donor levels has been revealed as a particularly noticeable effect in colloidal quantum dots as they are usually synthesized in media with a dielectric response rather different to that of the dot material. ${ }^{26,29,30}$

Prompted by the important role of coupled impurities in nanotechnology, we present in this paper a theoretical study on the molecular properties of a $D_{2}^{+}$system confined in a spherical quantum dot. We focus on the influence of both the QD size and the dielectric mismatch on the radiative lifetime, the bonding-antibonding energy splitting, and the electron charge distributions in the lowest-lying $D_{2}^{+}$molecular states, and analyze the dependence of the results on the relative position of the donors inside the quantum dot.

The paper is organized as follows. In Sec. II, we introduce the model employed in the description of the confined double donor system and outline the details of the numerical procedure used to solve the resulting Hamiltonian. The effects of spatial and dielectric confinements on the double donor system are presented and discussed in Sec. III. Conclusions, given in Sec. IV, end the paper.

\section{MODEL AND THEORETICAL OUTLINE}

The present study is carried out within the framework of the effective-mass and envelope function approximations. Consequently, we employ a macroscopic treatment of Coulombic interactions, and use a parameter, the dielectric con- 


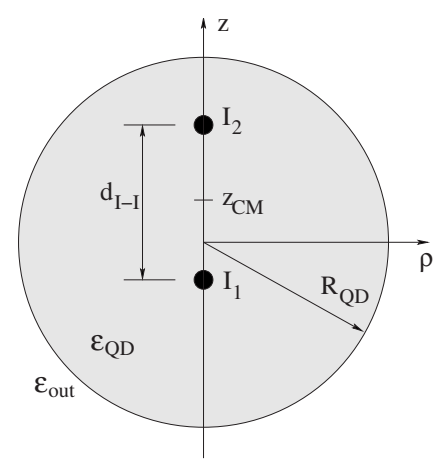

FIG. 1. Schematic representation of the system under study: an electron bound to two shallow donors separated by a distance $d_{I-I}$ within a diameter of a confining spherical QD with radius $R_{\mathrm{QD}}$ and dielectric constant $\varepsilon_{\mathrm{QD}}$ surrounded by a dielectric medium of permittivity $\varepsilon_{\text {out }} \cdot z_{\mathrm{CM}}$ stands for the center of mass of the coupled donors.

stant, to characterize the dielectric response of each material involved. The validity of such a treatment has been well established for both doped and undoped semiconductor heterostructures. ${ }^{31}$ Our model consists of a shallow donor pair confined in a spherical quantum dot with dielectric constant $\varepsilon_{\mathrm{QD}}$, which is in turn immersed in a dielectric medium with permittivity $\varepsilon_{\text {out }}$ (see Fig. 1). The corresponding effective-mass Hamiltonian for the bound conduction-band electron reads, in atomic units,

$$
H=-\frac{1}{2} \nabla\left(\frac{1}{m^{*}(\mathbf{r})} \nabla\right)+V(\mathbf{r})+\phi_{s}(\mathbf{r})+\sum_{i=1,2} \phi_{c}^{I_{i}}\left(\mathbf{r}, \mathbf{r}_{i}\right) .
$$

The first term on the right-hand side of Eq. (1) is the generalized kinetic-energy operator, ${ }^{32}$ which accounts for different electron masses in different materials. $V(\mathbf{r})$ is a steplike function representing the finite spatial confining potential due to the conduction-band offset between the media involved. $\phi_{c}^{I_{i}}\left(\mathbf{r}, \mathbf{r}_{i}\right)$ stands for the Coulomb potential generated by the impurity located at the fixed position $\mathbf{r}_{i}$, including the effect of the polarization charges induced at the dot surface as a consequence of the dielectric mismatch between the QD and the surrounding medium. The electron itself also induces polarization charges at the dot boundaries, whose effects are described by the self-polarization potential $\phi_{s}(\mathbf{r})$. When (as is the case here) a $\varepsilon(\mathbf{r})=\varepsilon(r)$ steplike dielectric function is assumed, $\phi_{c}$ and $\phi_{s}$ admit analytical expressions. ${ }^{33}$

In order to reduce the number of variables in the study, we fix the QD material parameters close to those of $\mathrm{CdS}\left(\varepsilon_{\mathrm{QD}}\right.$ $=5.5$ and $m^{*}=0.15$ ) (Ref. 34) unless otherwise indicated. Since we are not dealing with a specific surrounding medium, we will assume a typical depth of $V_{0}=4 \mathrm{eV}$ for the QD confining well and an effective mass $m^{*}=1$ for the external medium. Likewise, we will concentrate on donors which are aligned along an axis of the QD (see Fig. 1), although the conclusions obtained make possible to envisage the trends characterizing other configurations. This facilitates the calculation since the axial symmetry of the system allows

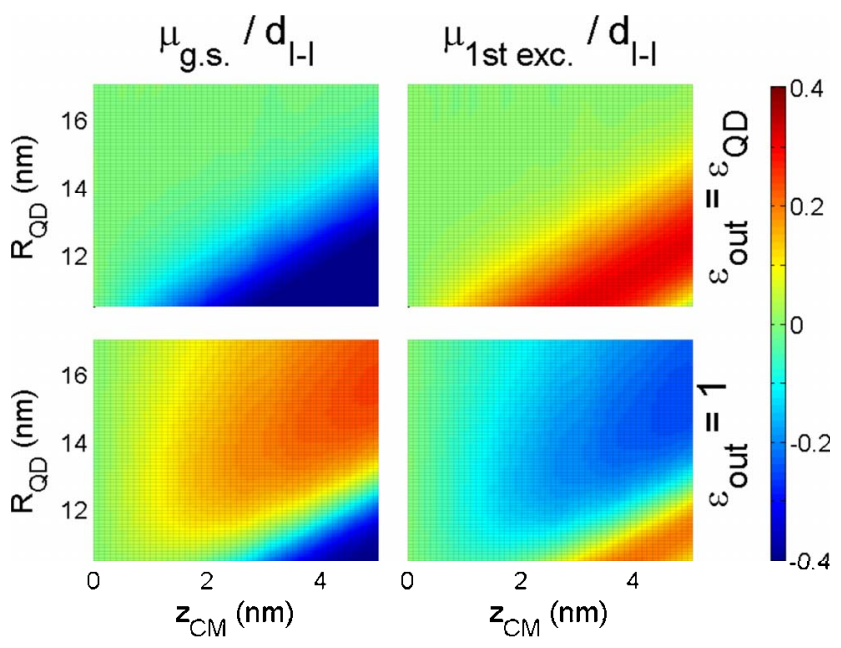

FIG. 2. (Color online) Dipolar moment [Eq. (2)] of the electron charge distribution for a confined $D_{2}^{+}$system with $d_{I-I}=10 \mathrm{~nm}$ as a function of $R_{\mathrm{QD}}$ and the system location in the dot. Left (right) panels correspond to the electron in the ground (first excited) state. In top (bottom) panels the QD is embedded in a $\varepsilon_{\text {out }}=\varepsilon_{\mathrm{QD}}\left(\varepsilon_{\text {out }}\right.$ $=1$ ) dielectric medium. The origin has been located at the QD center.

us to integrate analytically the angular coordinate of the electron in Eq. (1). Then, to obtain electron energies and wave functions, we carry out an exact (numerical) integration of $(\rho, z)$ coordinates by means of a discretization scheme based on the finite differences method. The explicit expressions of $\phi_{c}$ and $\phi_{s}$ together with a detailed description of the integration method can be found in Ref. 28.

\section{RESULTS}

We start by investigating the effect of the spatial and dielectric confinements on the electron charge distribution of the double donor lowest-lying states. When the center of mass of the donor pair is not located at the dot center $\left(z_{\mathrm{CM}}\right.$ $\neq 0$, see Fig. 1), the asymmetry of the QD confinement is expected to break the inversion symmetry of these states. To monitor (and quantify) the symmetry breakdown, we calculate the dipolar moment of the corresponding electron charge distributions with respect to $z_{\mathrm{CM}}$,

$$
\mu=\left\langle i\left|\mathbf{r}-z_{\mathrm{CM}} \mathbf{u}_{z}\right| i\right\rangle,
$$

where $i=1,2$ labels the ground and first excited states of the system. The results are shown in Fig. 2, where $\mu / d_{I-I}$ (with $\left.d_{I-I}=10 \mathrm{~nm}\right)$ is represented for these states as a function of the QD radius and the donors location within the dot. Calculations have been performed for two different surrounding dielectric constants, namely, $\varepsilon_{\text {out }}=\varepsilon_{\mathrm{QD}}$ (absence of dielectric confinement) and $\varepsilon_{\text {out }}=1$ (spatial and dielectric confinements). In the figure, positive values of $\mu / d_{I-I}$ indicate that the electron is distributed to a greater extent around the donor closer to the QD border, whereas negative values indicate its preference for the innermost impurity. From the $\varepsilon_{\text {out }}=\varepsilon_{\mathrm{QD}}$ panels we can observe that the spatial confinement favors the localization of the ground state in the inner donor 
and the excited state in the outer one. This trend, more pronounced for low QD radii and large $z_{\mathrm{CM}}$ values, is a consequence of the larger destabilization that the potential barrier produces in the surroundings of the outermost impurity. To minimize its energy, the electron in the ground state localizes in the inner donor and, to preserve orthogonality, the excited state concentrates in the outer one. In other words, nonsymmetric spatial confinement brings the homopolar $D_{2}^{+}$system to behave as heteropolar, leading to the spatial separation of the two lowest-lying electron states.

In the case $\varepsilon_{\text {out }}=1$ (lower panels in Fig. 2), we can see that, except for very small dot radii and very large $z_{\mathrm{CM}}$ values, the polarity of the states is opposite to that in the absence of dielectric confinement. Now, the ground state localizes the electron density in the outer donor, whereas the inner donor is occupied by the excited state. This effect can be explained as originated by the contribution of the polarization charges induced by the impurities at the QD surface. Impurity off-centering increases the nonhomogeneity of the corresponding induced charge distribution at the dot surface, this lack of homogeneity being responsible for an additional stabilization of off-centered electron locations. ${ }^{35}$ As a consequence, the total Coulomb potential is deeper at the position of the donor closer to the surface, so that the ground state distributes preferably around it. Therefore, spatial and dielectric confinements yield opposite spatial separation of the electron states ${ }^{36}$ and their interplay will determine the final configuration of the corresponding electron charge distributions in each case.

For a more detailed description of this interplay, in Fig. 3(a) we have depicted $\mu / d_{I-I}$ for the same system as in Fig. $2\left(d_{I-I}=10 \mathrm{~nm}\right)$ but for the particular case of $z_{\mathrm{CM}}=5 \mathrm{~nm}$ and a more extended $R_{\mathrm{QD}}$ range. For this value of $z_{\mathrm{CM}}$, one of the impurities is located at the QD center, a situation a priori easier to be obtained experimentally than any other. In the figure, solid (dashed) curves correspond to the ground (first excited) state. Interestingly, the case of pure spatial confinement ( $\varepsilon_{\text {out }}=\varepsilon_{\mathrm{QD}}$, thin red lines) shows homopolarity for large $R_{\mathrm{QD}}$ values, whereas in the presence of dielectric mismatch $\left(\varepsilon_{\text {out }}=1\right.$, thick blue lines) the system behaves as heteropolar. This is a signature of the different length scales in which spatial and dielectric confinements operate and is a consequence of the long range of image charge Coulomb interactions. The different length scales of these confinement sources were already reported by Goldoni et al., ${ }^{37}$ who also introduced the concept of remote dielectric tailoring to tune at will exciton-binding energies in semiconductor/insulator hybrid nanostructures.

For intermediate values of $R_{\mathrm{QD}}$ [see e.g., $R_{\mathrm{QD}} \approx 14 \mathrm{~nm}$ in Fig. 3(a)], where both spatial and dielectric confinement effects are significant, we can observe that the dielectric confinement compensates, and even inverts, the trend imposed by the spatial confinement. Finally, for low $R_{\mathrm{QD}}$ values, the spatial confinement dominates and the inner donor is preferred by the ground state in both cases.

The spatial separation of the two lowest-lying electron states is more pronounced as the interdonor distance increases. This is apparent in Fig. 4(a), which corresponds to the same system as in Fig. 3 (with one of the impurities at the dot center) but for $d_{I-I}=15 \mathrm{~nm}$. In this case, $\mu / d_{I-I}$ is
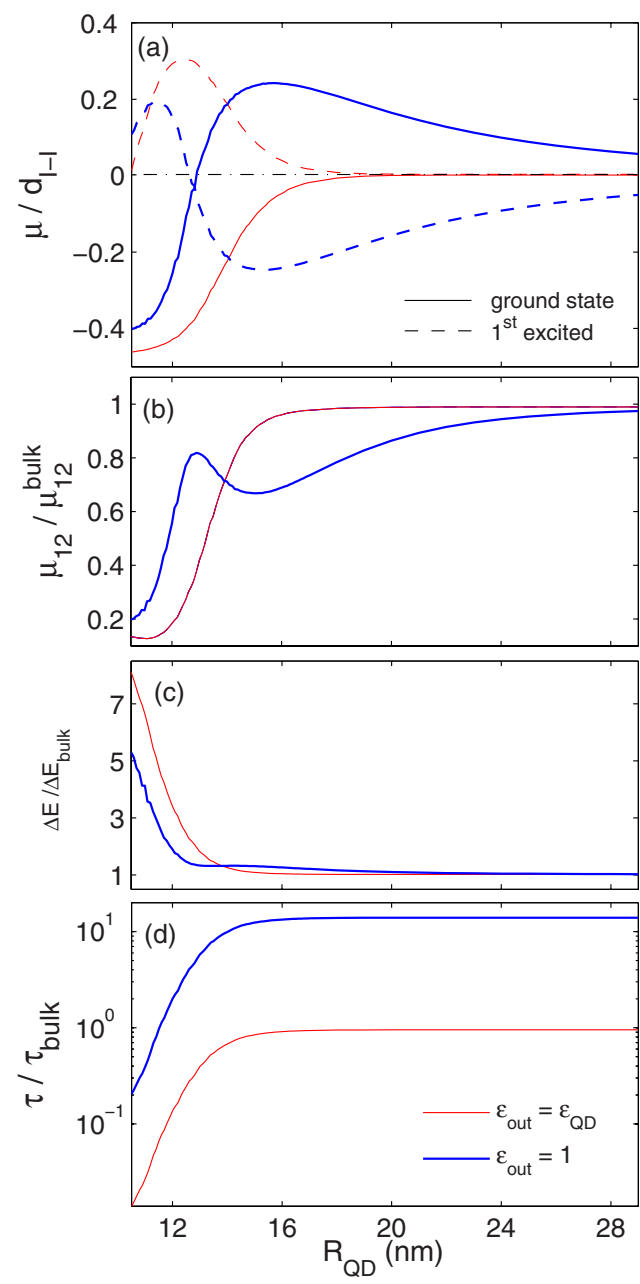

FIG. 3. (Color online) (a) Dipolar moment [Eq. (2)] of the electron charge distribution in the ground (solid lines) and first excited (dashed lines) states of the confined coupled donor system $\left(d_{I-I}\right.$ $=10 \mathrm{~nm}$ ) with one of the impurities located at the QD center as a function of the QD radius. Thick blue lines correspond to $\varepsilon_{\text {out }}=1$, whereas thin red lines correspond to $\varepsilon_{\text {out }}=\varepsilon_{\mathrm{QD}}$ (absence of dielectric confinement). (b) Corresponding transition moments, (c) splitting energies, and (d) first excited-state radiative lifetimes relative to bulk values.

plotted as a function of $\varepsilon_{\text {out }}$ for two different dot radii, namely, $R_{\mathrm{QD}}=20$ and $23 \mathrm{~nm}$. Again, solid and dashed lines are used for the ground and first excited states. As can be observed from the $R_{\mathrm{QD}}=20 \mathrm{~nm}$ curves, when $\varepsilon_{\text {out }}>\varepsilon_{\mathrm{QD}}$ the dielectric confinement magnifies the states spatial separation imposed by the spatial confinement $\left(\varepsilon_{\text {out }}=5.5\right)$. On the contrary, this trend is inverted as $\varepsilon_{\text {out }}$ diminishes. The bulklike homopolar character is recovered for a given value of $\varepsilon_{\text {out }}$ (3.9 in this case), compensating the effect of the spatial confinement. Finally, dielectric mismatch effects dominate for low enough values of $\varepsilon_{\text {out }}$, yielding again a spatial separation of the lowest-lying molecular states but with opposite polarity.

This evolution can be seen in Fig. 4(d), where the modulus of the wave function $\Psi(\mathbf{r})$ restricted to the azimuthal angle $\phi=0$ is represented for both the ground and first excited states and three different values of the surroundings 

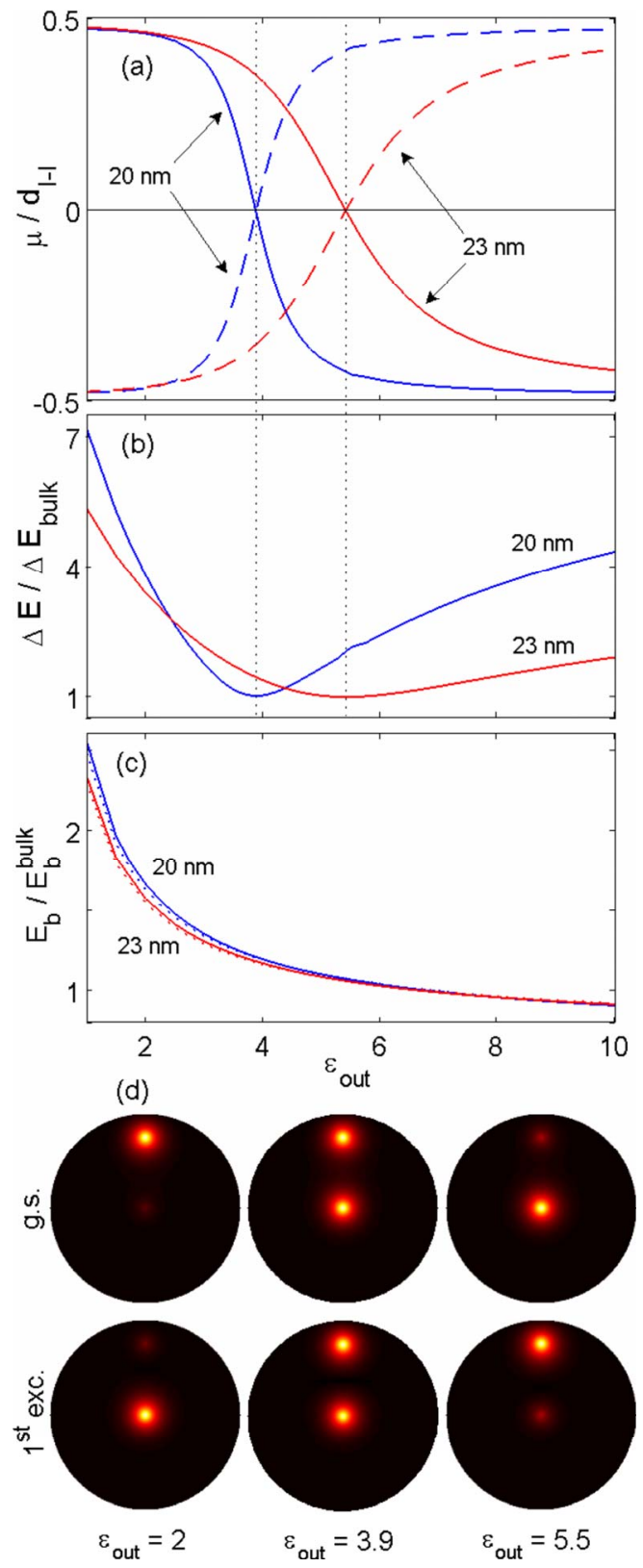

FIG. 4. (Color online) (a) Ground state (solid lines) and first excited-state (dashed lines) dipolar moments for a $d_{I-I}=15 \mathrm{~nm}$ confined $D_{2}^{+}$system with one donor at the QD center as a function of $\varepsilon_{\text {out }}$. Curves are shown for two different QD radii. (b) Splitting energy dependence on $\varepsilon_{\text {out }}$ for the two QD radii studied. (c) Corresponding ground-state binding energies (solid lines). Curves corresponding to $z_{\mathrm{CM}}=0$ (dotted lines) have been included for comparison. (d) Modulus of the wave function $\Psi(\mathbf{r})$ restricted to the azimuthal angle $\phi=0$ for the same system as above with $R_{\mathrm{QD}}$ $=20 \mathrm{~nm}$ and different values of $\varepsilon_{\text {out }}$.

permittivity, i.e., $\varepsilon_{\text {out }}=5.5$ (absence of dielectric confinement), $\varepsilon_{\text {out }}=3.9$ (situation in which the homopolar character is recovered), and $\varepsilon_{\text {out }}=2$ (a typical value of organic capping materials ${ }^{30}$ ).

The case shown in Fig. 4 for $R_{\mathrm{QD}}=23 \mathrm{~nm}$ represents an additional example of the long range of dielectric effects. While in this case bulk charge distributions are hardly affected by the QD spatial confinement, the proper choice of the QD environment can still yield important changes in the relative electronegativity of the donors and, therefore, in the electron charge distributions.

The energy splitting $(\Delta E)$ between the ground and first excited states is also influenced by dielectric and spatial confinements [see Fig. 4(b)]. As derived from the figure, $\Delta E$ increases as the spatial separation of the states is more pronounced, presenting a minimum, close to the bulk value, when homopolar conditions are tuned. Contrary to $\Delta E$, the corresponding binding energies ${ }^{38}\left(E_{b}\right)$ are negligibly correlated with the spatial separation of the states [see Fig. 4(c)]. This can be derived from the comparison of solid and dotted curves in the figure, which correspond to $z_{\mathrm{CM}}=7.5 \mathrm{~nm}$ (one donor at the QD center) and $z_{\mathrm{CM}}=0$ (symmetric confinement), respectively. Indeed, though both cases present similar $E_{b}$ values, for $z_{\mathrm{CM}}=0$ the system presents homopolarity regardless of $\varepsilon_{\text {out }}$, whereas this is not the case for $z_{\mathrm{CM}}$ $=7.5 \mathrm{~nm}$. Therefore, $E_{b}$ is negligibly influenced by the offcentering of the $D_{2}^{+}$system and then by the spatial separation of the states. Conversely, as expected, $E_{b}$ is strongly affected by the dielectric environment, undergoing a large increase as $\varepsilon_{\text {out }}$ decreases.

It is well known that the spatial separation of logic states in charge-based qubit realizations can efficiently reduce the decoherence of the charge degrees of freedom coding the qubit, which makes possible to perform the coherent quantum logic operations at a fairly high ratio. ${ }^{8}$ Therefore, confinement is expected to affect coherence in our system. In donor-based charge qubits, decoherence caused by interaction with phonons occurs in time scales that can be made much longer than all other time scales in the problem by choosing an appropriate donor separation. ${ }^{20}$ Then, to study how confinement affects decoherence in our system, we will neglect in a first approximation acoustic and optical phonon scattering and will only take into account decoherence coming from vacuum electromagnetic fluctuations (radiative decay).

The radiative decay rate in a spherical quantum dot can be estimated within the dipole approximation by ${ }^{39}$

$$
\tau^{-1}=\frac{9 \varepsilon_{\text {out }}^{5 / 2}}{\left(2 \varepsilon_{\text {out }}+\varepsilon_{\mathrm{QD}}\right)^{2}} \frac{\Delta E^{3}}{3 \pi \varepsilon_{0} \hbar^{4} c^{3}} \mu_{12},
$$

where $c$ is the speed of light in vacuum, $\varepsilon_{0}$ is the vacuum dielectric constant, $\tau$ is the radiative lifetime, and $\mu_{12}$ $=|\langle 1|\mathbf{r}| 2\rangle|^{2}$ is the square of the dipole transition moment between the states $|1\rangle$ and $|2\rangle$, which are in our case the ground and first excited states of the system.

$\mu_{12}$, relative to its value in the absence of spatial and dielectric confinements, is depicted in Fig. 3(b) for the studied system. Again, results for $\varepsilon_{\text {out }}=\varepsilon_{\mathrm{QD}}$ and $\varepsilon_{\text {out }}=1$ are shown. As can be seen in the figure, $\mu_{12}$ decreases as the system moves away from homopolar conditions. By inspection of Eq. (3), one could expect that a decrease in $\mu_{12}$ would help in increasing the lifetime. However, the dependence of $\tau^{-1}$ on $\Delta E^{3}$ cannot be neglected in this case since, as we showed before, spatial separation of the two lowest-lying states is paralleled by an increase in the transition energy [see Fig. 3(c)]. On balance, the influence of $\Delta E$ on the radia- 
CdS

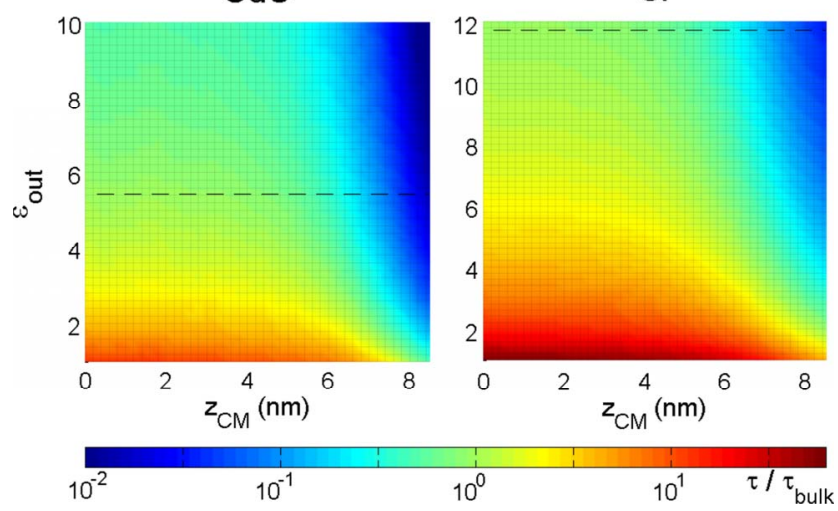

FIG. 5. (Color online) First excited-state radiative lifetime (relative to bulk) for a $d_{I-I}=10 \mathrm{~nm} D_{2}^{+}$system confined in a $R$ $=14 \mathrm{~nm}$ QD as a function of $\varepsilon_{\text {out }}$ and the system location within the QD. Host material parameters are similar to those of CdS (left) and Si (right). Dashed lines indicate the absence of dielectric confinement in each case.

tive lifetime is larger than that of $\mu_{12}$. This can be seen in Fig. 3(d), where $\tau$ has been represented relative to $\tau_{\text {bulk. }}$. Referring to the results for $\varepsilon_{\text {out }}=\varepsilon_{\mathrm{QD}}$ (where only the spatial confinement is influencing the system), we observe that $\tau$ drops strongly for small values of $R_{\mathrm{QD}}$, whereas it reaches the bulk value when the spatial separation of the two lowestlying states disappears and homopolar conditions are recovered.

The results for $\varepsilon_{\text {out }}=1$ are more interesting. $\tau$ shows a profile similar to that of $\varepsilon_{\text {out }}=\varepsilon_{\mathrm{QD}}$ calculations but despite spatial separation still persists in the whole range of $R_{\mathrm{QD}}$ under study, $\tau$ reaches values more than 1 order of magnitude larger than in bulk. The enhancement of $\tau$ when the QD is surrounded by a low-dielectric constant medium is a consequence of the influence of local-field effects, ${ }^{39}$ which have been observed experimentally through measurements of exciton recombination rates in QDs embedded in apolar solvents with low refractive indices. ${ }^{30}$

The drop of $\tau$ for small values of $R_{\mathrm{QD}}$ in Fig. 3(d) is induced, in both represented cases, by the spatial confinement through the large increase that it yields on $\Delta E$. This can be confirmed in Fig. 5 (left panel) where the radiative lifetime of our system as a function of $\varepsilon_{\text {out }}$ and the system location within a $R=14 \mathrm{~nm}$ QD is shown. When the off centering of the donor pair is not much pronounced (low and medium $z_{\mathrm{CM}}$ values), the influence of the spatial confinement is negligible and, in the absence of dielectric mismatch, $\tau$ keeps close to the bulk value. In these conditions, $\tau$ increases noticeably with respect to the bulk as $\varepsilon_{\text {out }}$ decreases. However, as the donor pair gets closer to the dot surface (large $z_{\mathrm{CM}}$ values), the radiative lifetime becomes dominated by the effects of spatial confinement. $\tau$ experiences a strong reduction, which is only partially compensated by decreasing $\varepsilon_{\text {out }}$.

Finally, to probe the effect of increasing the QD dielectric constant, we have repeated the calculations for QD parameters similar to those of $\mathrm{Si}\left(\varepsilon_{\mathrm{QD}}=11.7\right.$ and $\left.m^{*}=0.26\right)$. The results, shown in the right panel of Fig. 5, evidence that in this case $\tau$ may increase up to almost 2 orders of magnitude with respect to the corresponding bulk value.

\section{CONCLUDING REMARKS}

We have probed the influence of spatial and dielectric confinements on spontaneous emission rates, energy splittings, and charge-density distributions in the lowest-lying states of a $D_{2}^{+}$double donor system confined in a spherical quantum dot. We found that asymmetric confinement (produced when the donors are not symmetrically distributed around the QD center) leads the otherwise homopolar donor pair to behave as heteropolar, diminishing the interdonor tunnel coupling and localizing the ground and first excited states in different donors. In this regard, when (as usual) $\varepsilon_{\text {out }}$ $<\varepsilon_{\mathrm{QD}}$, spatial confinement tends to localize the ground/first excited state in the inner/outer donor, whereas dielectric effects tend to distribute them oppositely. However, these confinement sources operate in different length scales. While in the large dot size regime only dielectric effects influence the $D_{2}^{+}$system, in the small dot size regime spatial confinement effects dominate. This makes possible to tune the tunnel coupling strength and charge-density distribution in the $D_{2}^{+}$system at will, by choosing the appropriate dot size and surrounding medium.

We also found that bulk radiative lifetimes are strongly reduced by spatial confinement due to the enhanced splitting energies. On the other hand, when spatial confinement effects are no longer involved (large dot sizes), radiative lifetimes may increase orders of magnitude when the QDs are embedded in low-dielectric constant media.

Summing up, the confinement of the $D_{2}^{+}$system in a quantum dot provides additional mechanisms to mold its properties; it is worth to notice the spatial distribution of the lowest-lying states, the corresponding energy splittings, and the radiative lifetimes. The (wide range) tuning capability of this last property could be of interest to increase the relatively low bulk coherence times of orbital states in charge qubit realizations.

We close by stressing that confinement might also be useful to improve the performance of optically driven donorbased charge qubit schemes. ${ }^{17,18}$ In such schemes, the decoherence caused by the ionization and spontaneous emission of the excited states acting as connecting channels between the logical states could be minimized through the proper choice of QD size and dielectric environment. On one hand, quantum confinement would lift the quasidegeneracy among near-continuum states, increasing the corresponding ionization threshold and excitation selectivity. On the other hand, the employment of low dielectric constant surrounding materials would help in reducing their (otherwise limiting) spontaneous emission rates.

\section{ACKNOWLEDGMENTS}

Support from MICINN under Project No. CTQ200803344 and Bancaixa under Project No. P1-1B2006-03 is acknowledged. 
*josep.planelles@qfa.uji.es

${ }^{1}$ S. A. Wolf, D. D. Awschalom, R. A. Buhrman, J. M. Daughton, S. von Molnár, M. L. Roukes, A. Y. Chtchelkanova, and D. M. Treger, Science 294, 1488 (2001).

${ }^{2}$ Nano-optoelectronics: Concepts, Physics and Devices, edited by M. Grundmann (Springer-Verlag, Berlin, 2002).

${ }^{3}$ A. J. Nozik, Physica E (Amsterdam) 14, 115 (2002).

${ }^{4}$ B. Bayer, P. Hawrylak, K. Hinzer, S. Fafard, M. Korkusinski, Z. R. Wasilewski, O. Stern, and A. Forchel, Science 291, 451 (2001).

${ }^{5}$ E. A. Stinaff, M. Scheibner, A. S. Bracker, I. V. Ponomarev, V. L. Korenev, M. E. Ware, M. F. Doty, T. L. Reinecke, and D. Gammon, Science 311, 636 (2006).

${ }^{6}$ J. R. Petta, A. C. Johnson, C. M. Marcus, M. P. Hanson, and A. C. Gossard, Phys. Rev. Lett. 93, 186802 (2004).

${ }^{7}$ A. S. Bracker, M. Scheibner, M. F. Doty, E. A. Stinaff, I. V. Ponomarev, J. C. Kim, L. J. Whitman, T. L. Reinecke, and D. Gammon, Appl. Phys. Lett. 89, 233110 (2006).

${ }^{8}$ X. Q. Li and Y. Arakawa, Phys. Rev. A 63, 012302 (2000).

${ }^{9}$ J. L. O'Brien, S. R. Schofield, M. Y. Simmons, R. G. Clark, A. S. Dzurak, N. J. Curson, B. E. Kane, N. S. McAlpine, M. E. Hawley, and G. W. Brown, Phys. Rev. B 64, 161401(R) (2001).

${ }^{10}$ S. R. Schofield, N. J. Curson, M. Y. Simmons, F. J. Ruess, T. Hallam, L. Oberbeck, and R. G. Clark, Phys. Rev. Lett. 91, 136104 (2003).

${ }^{11}$ T. Shinada, S. Okamoto, T. Kobayashi, and L. Ohdomari, Nature (London) 437, 1128 (2005).

${ }^{12}$ R. J. Schoelkopf, P. Wahlgren, A. A. Hozhevnikov, P. Delsing, and D. E. Prober, Science 280, 1238 (1998).

${ }^{13}$ A. Aassime, G. Johansson, G. Wendin, R. J. Schoelkopf, and P. Delsing, Phys. Rev. Lett. 86, 3376 (2001).

${ }^{14}$ M. J. Calderón, B. Koiller, and S. Das Sarma, Phys. Rev. B 75, 125311 (2007).

${ }^{15}$ M. J. Calderón, B. Koiller, X. Hu, and S. Das Sarma, Phys. Rev. Lett. 96, 096802 (2006).

${ }^{16}$ L. C. L. Hollenberg, A. S. Dzurak, C. Wellard, A. R. Hamilton, D. J. Reilly, G. J. Milburn, and R. G. Clark, Phys. Rev. B 69, 113301 (2004).

${ }^{17}$ A. V. Tsukanov, Phys. Rev. B 76, 035328 (2007).

${ }^{18}$ L. A. Openov, Phys. Rev. B 70, 233313 (2004).

${ }^{19}$ B. Koiller, X. Hu, and S. Das Sarma, Phys. Rev. B 73, 045319 (2006).

${ }^{20}$ S. D. Barrett and G. J. Milburn, Phys. Rev. B 68, 155307 (2003).

${ }^{21}$ F. H. L. Koppens, C. Buizert, K. J. Tielrooij, I. T. Vink, K. C. Nowack, T. Meunier, L. P. Kouwenhoven, and L. M. K. Vander- sypen, Nature (London) 442, 766 (2006).

${ }^{22}$ B. Li, B. Partoens, F. M. Peeters, and W. Magnus, Phys. Rev. B 79, 085306 (2009); Microelectron. J. 40, 446 (2009).

${ }^{23}$ S. Kang, Y. M. Liu, and T. Y. Shi, Eur. Phys. J. B 63, 37 (2008).

${ }^{24}$ S. Kang, Y. M. Liu, and T. Y. Shi, Commun. Theor. Phys. 50, 767 (2008)

${ }^{25}$ X. Hu and S. Das Sarma, Phys. Rev. A 61, 062301 (2000).

${ }^{26}$ R. Tsu and D. Babić, Appl. Phys. Lett. 64, 1806 (1994).

${ }^{27}$ M. Lannoo, C. Delerue, and G. Allan, Phys. Rev. Lett. 74, 3415 (1995); G. Allan, C. Delerue, M. Lannoo, and E. Martin, Phys. Rev. B 52, 11982 (1995); J. M. Ferreyra, P. Bosshard, and C. R. Proetto, ibid. 55, 13682 (1997); B. Li, A. F. Slachmuylders, B. Partoens, W. Magnus, and F. M. Peeters, ibid. 77, 115335 (2008).

${ }^{28}$ J. L. Movilla and J. Planelles, Phys. Rev. B 71, 075319 (2005); Comput. Phys. Commun. 170, 144 (2005).

${ }^{29}$ M. Bruchez, Jr., M. Moronne, P. Gin, S. Weiss, and A. P. Alivisatos, Science 281, 2013 (1998); R. Tsu, Microelectron. J. 34, 329 (2003).

${ }^{30}$ S. F. Wuister, C. M. Donegá, and A. Meijerink, J. Chem. Phys. 121, 4310 (2004).

${ }^{31}$ C. Delerue, M. Lannoo, and G. Allan, Phys. Rev. B 68, 115411 (2003); X. Cartoixà and L. W. Wang, Phys. Rev. Lett. 94, 236804 (2005); D. Ninno, F. Trani, G. Cantele, K. J. Hameeuw, G. Iadonisi, E. Degoli, and S. Ossicini, Europhys. Lett. 74, 519 (2006).

${ }^{32}$ D. BenDaniel and C. B. Duke, Phys. Rev. 152, 683 (1966); P. Harrison, Quantum Wells, Wires and Dots (Wiley, Chichester, 2001).

${ }^{33}$ P. G. Bolcatto and C. R. Proetto, J. Phys.: Condens. Matter 13, 319 (2001).

${ }^{34}$ A. D. Yoffe, Adv. Phys. 50, 1 (2001).

${ }^{35} \mathrm{~A}$ thorough analysis of the dependence of the polarization potentials on the impurities off-centering can be found in Ref. 28.

${ }^{36}$ This is true as long as $\varepsilon_{\text {out }}<\varepsilon_{\mathrm{QD}}$ (the usual situation). When $\varepsilon_{\text {out }}>\varepsilon_{\mathrm{QD}}$, both sources of confinement favor the localization of the ground state in the inner donor.

${ }^{37}$ G. Goldoni, F. Rossi, and E. Molinari, Phys. Rev. Lett. 80, 4995 (1998).

${ }^{38}$ The binding energy has been calculated as the difference between the electron ground-state energies in the absence and in the presence of the impurities.

${ }^{39}$ A. Thränhardt, C. Ell, G. Khitrova, and H. M. Gibbs, Phys. Rev. B 65, 035327 (2002); R. J. Glauber and M. Lewenstein, Phys. Rev. A 43, 467 (1991). 